\title{
A positive role for polycomb in transcriptional regulation via H4K20me1
}

Xiangdong Lv ${ }^{1}$, Zhijun $\mathrm{Han}^{2}$, Hao Chen ${ }^{1}$, Bo Yang ${ }^{1}$, Xiaofeng Yang ${ }^{1}$, Yuanxin Xia ${ }^{1}$, Chenyu Pan ${ }^{1}$, Lin Fu ${ }^{1}$, Shuo Zhang ${ }^{1}$, Hui Han ${ }^{1}$, Min Wu ${ }^{3}$, Zhaocai Zhou ${ }^{1}$, Lei Zhang ${ }^{1,5}$, Lin $\mathrm{Li}^{4,5}$, Gang Wei ${ }^{2}$, Yun Zhao,

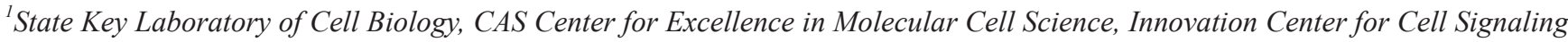
Network, Institute of Biochemistry and Cell Biology, Shanghai Institutes for Biological Sciences, Chinese Academy of Sciences, Shanghai 200031, China; ${ }^{2}$ Key Laboratory of Computational Biology, CAS-MPG Partner Institute for Computational Biology, Shanghai Institutes for Biological Sciences, Chinese Academy of Sciences, Shanghai 200031, China; ${ }^{3}$ Department of Biochemistry and Molecular Biology, College of Life Sciences and Key Laboratory of Intestinal and Colorectal Diseases, Wuhan University, Wuhan, Hubei 430072, China; ${ }^{4}$ State Key Laboratory of Molecular Biology, CAS Center for Excellence in Molecular Cell Science, Innovation Center for Cell Signaling Network, Institute of Biochemistry and Cell Biology, Shanghai Institutes for Biological Sciences, Chinese Academy of Sciences, Shanghai 200031, China; ${ }^{5}$ School of Life Science and Technology, Shanghai Tech University, Shanghai 200031, China

Cell Research (2017) 27:594. doi:10.1038/cr.2017.50; published online on 7 April 2017

Correction to: Cell Research (2016) 26:529-542. doi:10.1038/cr.2016.33; published online on 22 March 2016

The authors apologized for the missing of labeling the first two authors as co-first authors, since they contributed equally to this work. The correct form is as follows. We are also sorry for the delayed notice.

Xiangdong $\mathrm{Lv}^{1,}$, Zhijun Han ${ }^{2, *}$, Hao Chen ${ }^{1}$, Bo Yang ${ }^{1}$, Xiaofeng Yang ${ }^{1}$, Yuanxin Xia ${ }^{1}$, Chenyu Pan ${ }^{1}$, Lin Fu ${ }^{1}$, Shuo Zhang ${ }^{1}$, Hui Han ${ }^{1}$, Min Wu ${ }^{3}$, Zhaocai Zhou ${ }^{1}$, Lei Zhang ${ }^{1,5}$, Lin $\mathrm{Li}^{4,5}$, Gang Wei ${ }^{2}$, Yun Zhao ${ }^{1,5}$

*These two authors contributed equally to this work. 